\title{
Canonical and 1-deoxy(methyl) sphingoid bases: tackling the effect of lipid structure on membrane biophysical properties.
}

Tania C.B. Santos ${ }^{\dagger, \ddagger}$, Alexandra Vaz ${ }^{\dagger}$, Ana E. Ventura ${ }^{\dagger \neq}$, Essa M. Saied ${ }^{\S, \|}$, Christoph Arenz $z^{\S}$ Aleksander Fedorov ${ }^{\ddagger}$, Manuel Prieto ${ }^{\ddagger}$, Liana C. Silva ${ }^{\dagger} *$.

tResearch Institute for Medicines (iMed.ULisboa), Faculty of Pharmacy, Universidade de Lisboa, Lisbon, Portugal;

$\neq_{\mathrm{iBB}}$ - Institute for Bioengineering and Biosciences, Instituto Superior Técnico, Universidade de Lisboa, Lisboa, Portugal

§Humboldt Universität zu Berlin, Institute for Chemistry, Berlin, Germany.

"Suez Canal University, Faculty of Science, Chemistry Department, Ismailia, Egypt.

* corresponding author: lianacsilva@ff.ulisboa.pt

No of pages: 3

No of figures: 3 

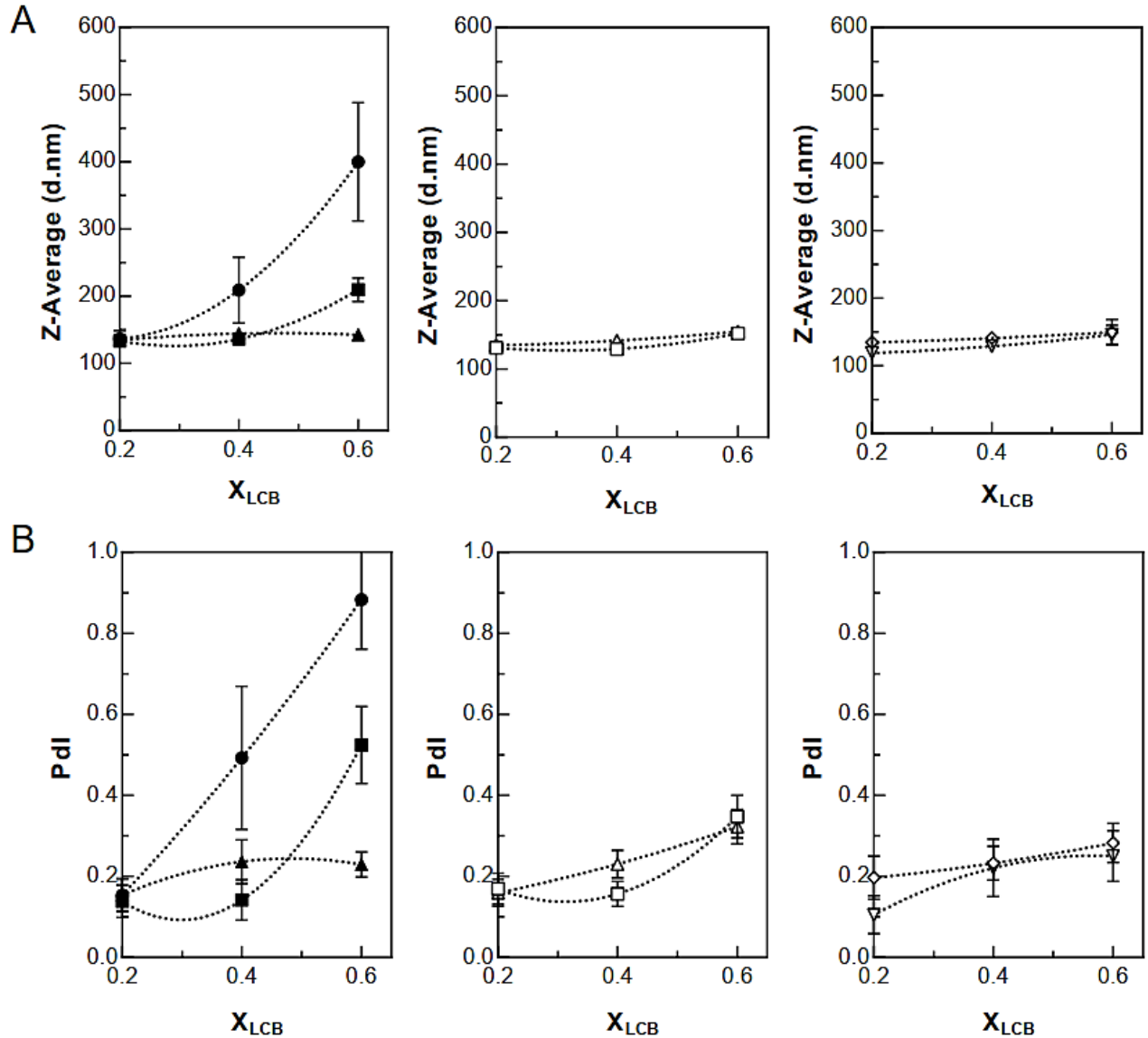

Figure S1. Hydrodynamic diameters measurements of POPC vesicles in the presence of canonical and 1-deoxy(methyl)-LCBs. (A) Average diameter and (B) PdI of binary mixtures of POPC with SA (circles), deoxy-SA (black squares), deoxymethyl-SA (black triangles), deoxy-Sph ${ }^{\Delta 4 t}$ (open squares), deoxymethyl-Sph ${ }^{\Delta 3 t}$ (open triangles), deoxy-Sph ${ }^{\Delta 14 c}$ (diamonds) or deoxymethyl-Sph ${ }^{\Delta 13 c}$ (inverted triangles) as a function of LCB molar fraction. Values are means \pm SD of at least three independent experiments. The lines act merely as guides to the eye. Samples with PdI values $\leq 0.1$ are considered highly monodisperse, $0.1<\mathrm{PdI}<0.4$ are moderately polydisperse while samples with $\mathrm{PdI}>0.4$ are highly polydisperse $\mathrm{P}^{70}$. 

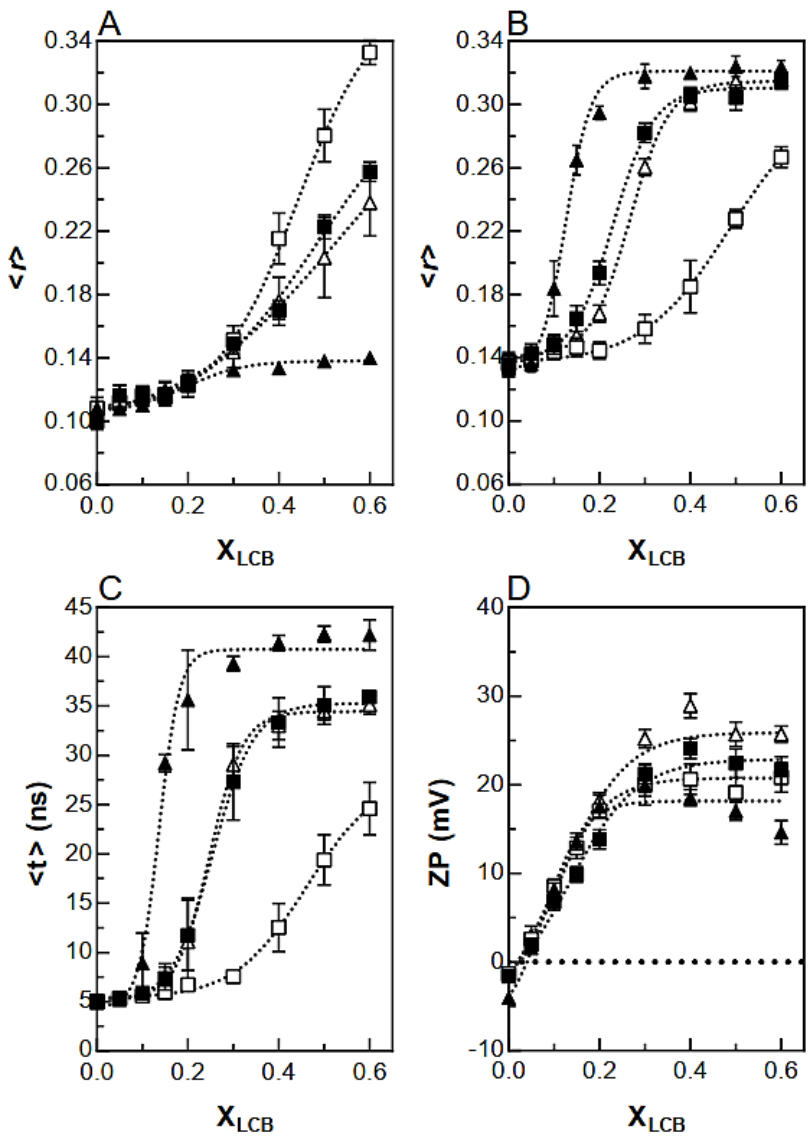

Figure S2. Effect of the presence of a trans double bond on deoxy (methyl)-LCBs on the fluidity of POPC membranes. (A,B) Steady state fluorescence anisotropy of (A) DPH and (B) $t$-PnA; (C) Mean fluorescence lifetime of $t$-PnA and (D) $\zeta$-potential of binary mixtures of POPC in the presence of saturated LCBs (solid symbols) or unsaturated LCBs (open symbols) as a function of LCB molar fraction. LCBs lacking the C1-hydroxyl group are represented as squares and LCBs lacking the C1-methoxy group are represented as triangles. Values are means \pm SD of at least three independent experiments. The lines act merely as guides to the eye.

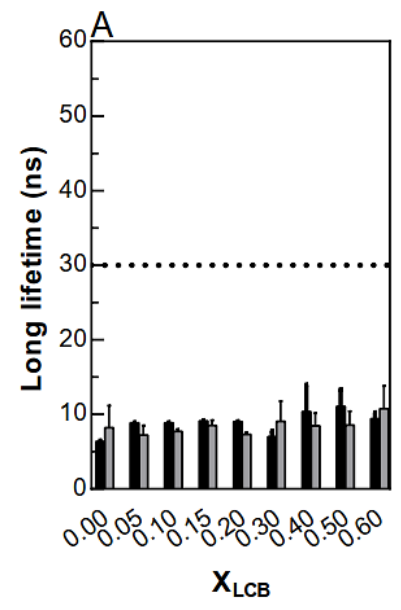

Figure S3. Fluorescence lifetime of $t$-PnA in deoxy-Sph ${ }^{\Delta 14 c}$ and deoxymethyl-Sph ${ }^{\Delta 13 c}$ vesicles. (A) Long lifetime component of $t$-PnA in binary mixtures of POPC with deoxy-Sph ${ }^{\Delta 14 c}$ (black bars) or deoxymethyl-Sph ${ }^{\Delta 13 c}$ (grey bars) as a function of LCB molar fraction. Values are means \pm SD of at least three independent experiments. 